\title{
Spinal fusion for pediatric neuromuscular scoliosis: national trends, complications, and in-hospital outcomes
}

\author{
Kavelin Rumalla, Chester K. Yarbrough, MD, MPHS, Andrew J. Pugely, MD, Linda Koester, BS, \\ and lan G. Dorward, MD
}

Department of Neurological Surgery, Washington University School of Medicine, St. Louis, Missouri

OBJECTIVE The objective of this study was to determine if the recent changes in technology, surgical techniques, and surgical literature have influenced practice trends in spinal fusion surgery for pediatric neuromuscular scoliosis (NMS). In this study the authors analyzed recent trends in the surgical management of NMS and investigated the effect of various patient and surgical factors on in-hospital complications, outcomes, and costs, using the Nationwide Inpatient Sample (NIS) database.

METHODS The NIS was queried from 2002 to 2011 using International Classification of Diseases, Ninth Edition, Clinical Modification codes to identify pediatric cases (age < 18 years) of spinal fusion for NMS. Several patient, surgical, and short-term outcome factors were included in the analyses. Trend analyses of these factors were conducted. Both univariate and multivariable analyses were used to determine the effect of the various patient and surgical factors on short-term outcomes.

RESULTS Between 2002 and 2011, a total of 2154 NMS fusion cases were identified, and the volume of spinal fusion procedures increased 93\% from 148 in 2002 to 286 in 2011 ( $p<0.0001)$. The mean patient age was $12.8 \pm 3.10$ years, and $45.6 \%$ of the study population was female. The overall complication rate was $40.1 \%$ and the respiratory complication rate was $28.2 \%$. From 2002 to 2011 , upward trends ( $p<0.0001$ ) were demonstrated in Medicaid insurance status ( $36.5 \%$ to $52.8 \%)$, presence of $\geq 1$ comorbidity ( $40.2 \%$ to $52.1 \%$ ), and blood transfusions $(25.2 \%$ to $57.3 \%$ ). Utilization of posterior-only fusions (PSFs) increased from $66.2 \%$ to $90.2 \%(p<0.0001)$ while combined anterior release/fusions and PSF (AR/PSF) decreased from 33.8\% to 9.8\% (<0.0001). Intraoperative neurophysiological monitoring (IONM) underwent increasing utilization from 2009 to 2011 (15.5\% to 20.3\%, $p<0.0001)$. The use/harvest of autograft underwent a significant upward trend between 2002 and 2011 (31.3\% to 59.8\%, p < 0.0001). In univariate analysis, IONM use was associated with decreased complications $(40.7 \%$ to $33.1 \%, p=0.049)$ and length of stay (LOS; 9.21 to 6.70 days, $p<0.0001$ ). Inflation-adjusted mean hospital costs increased nearly $75 \%$ from 2002 to 2011 ( $\$ 36,805$ to $\$ 65,244, p<$ 0.0001). In the multivariable analysis, nonwhite race, highest quartile of median household income, greater preexisting comorbidity, long-segment fusions, and use of blood transfusions were found to increase the likelihood of complication occurrence (all $p<0.05$ ). In further multivariable analysis, independent predictors of prolonged LOS included older age, increased preexisting comorbidity, the AR/PSF approach, and long-segment fusions (all $p<0.05$ ). Lastly, the likelihood of increased hospital costs (at or above the 90th percentile for LOS, 14 days) was increased by older age, female sex, Medicaid insurance status, highest quartile of median household income, AR/PSF approach, long-segment fusion, and blood transfusion (all $p<0.05$ ). In multivariable analysis, the use of autograft was associated with a lower likelihood of complication occurrence and prolonged LOS (both $p<0.05$ ).

CONCLUSIONS Increasing use of IONM and posterior-only approaches may combat the high complication rates in NMS. The trends of increasing comorbidities, blood transfusions, and total costs in spinal fusion surgery for pediatric NMS may indicate an increasingly aggressive approach to these cases.

http://thejns.org/doi/abs/10.3171/2016.2.SPINE151377

KEY WORDS neuromuscular scoliosis; spinal fusion; pediatric; trends; management; complications; outcomes; Kids Inpatient Database; deformity

ABBREVIATIONS AHRQ = Agency for Healthcare Research and Quality; $\mathrm{AR}=$ anterior release/fusion; $\mathrm{BMP}=$ bone morphogenetic protein; $\mathrm{Cl}=$ confidence interval; HCUP = Healthcare Cost and Utilization Project; ICD-9-CM = International Classification of Diseases, Ninth Edition, Clinical Modification; IONM = intraoperative neurophysiological monitoring; LOS = length of stay; NIS = Nationwide Inpatient Sample; NMS = neuromuscular scoliosis; OR = odds ratio; PSF = posterior-only spinal fusion. SUBMITTED November 23, 2015. ACCEPTED February 11, 2016.

INCLUDE WHEN CITING Published online May 20, 2016; DOI: 10.3171/2016.2.SPINE151377. 
$\mathrm{N}$ EUROMUSCULAR Scoliosis (NMS) is a type of spinal deformity resulting from underlying neurological pathology. ${ }^{4}$ The deformity of NMS is often severe, involving the entire thoracolumbar spine and pelvis, progressing rapidly, and resulting in visceral organ system dysfunction. Once scoliosis curves progress beyond a certain magnitude, surgical intervention remains the last reliable treatment option. Ultimately, the decision to proceed with surgery is based on curve progression, concomitant medical comorbidities, and patient quality of life. ${ }^{4,13}$ Complications after surgery for NMS occur more often and with greater severity than those for idiopathic scoliosis. ${ }^{1,36}$

Recent years have brought substantial advancements in preoperative optimization, surgical techniques and instrumentation, intraoperative monitoring, and control of perioperative and postoperative infection and complications. ${ }^{4,13}$ These advancements necessitate a reevaluation of appropriate surgical techniques and approaches. In the past decade, several studies have addressed the debate regarding a combined anterior/posterior fusion approach versus an all-posterior fusion approach, with a trend toward utilizing posterior-only approaches. . $^{2,11,26,27,31,33,39,40}$ The choice of surgical technique must take into account surgeon experience, curve progression, and concomitant medical comorbidities. ${ }^{1,4,13}$ Awareness of perioperative and postoperative in-hospital complications and outcomes associated with the surgical correction of NMS is important for patient counseling and surgical management decisions.

Given these shifts in technology and surgeon preferences, few studies have examined the longitudinal impact of these evolving trends on short-term complications, outcomes, and costs. With the emergence of large-scale national health care claims databases, such as the Nationwide Inpatient Sample (NIS), researchers are now able to answer important epidemiological questions. Thus, the purpose of this study was to examine recent trends in the surgical management of NMS and investigate the effect of perioperative characteristics on in-hospital complications, outcomes, and costs using national discharge data from the NIS.

\section{Methods \\ Data Source}

The NIS is the largest publicly available all-payer inpatient database in the US and is known to be one of the most valid and reliable data sources for epidemiological estimates involving inpatient care (http://www.hcup-us.ahrq. gov/nisoverview.jsp). The database is developed and maintained under the Healthcare Cost and Utilization Project (HCUP), a subsidiary of the federal government's Agency for Healthcare Research and Quality (AHRQ). The NIS provides annual admission and discharge data from a $20 \%$ stratified sample of all hospitals, excluding rehabilitation and long-term care hospitals. The NIS Trend Weights files are used to create national estimates for trend analyses to adjust for changes in the NIS over time (http://www.hcupus.ahrq.gov/db/nation/nis/trendwghts.jsp). Unique subject identifiers are used to conceal patient identity and prevent identification of multiple admissions for the same patient, thus the database is deemed compliant with the Health Insurance Portability and Accountability Act (HIPAA) and exempt from institutional review board approval.

\section{Inclusion Criteria}

We queried the NIS database from 2002 to 2011 to identify all patients 17 years of age or younger who had a diagnosis of NMS (International Classification of Diseases, Ninth Edition, Clinical Modification [ICD-9-CM] code 737.43).

Patients were included and analyzed further if they underwent spinal fusion surgery (ICD-9-CM primary procedure codes $81.02-81.08$ ). We excluded cases of primary revision surgery (codes 81.32-81.38), cases of spinal fusion levels of less than 4 vertebrae (code 81.62), and cases of nonfusion growing-rod lengthening. Records were excluded from the analysis if information for age, sex, or primary diagnosis was missing. We report the percentage of missing/unspecified records for other variables in Tables 1 and 2. Diagnostic and procedural information in the NIS are identified using the ICD-9-CM codes.

\section{Parameters of Interest}

Preoperative parameters of interest included demographics, hospital characteristics, and comorbidities. Demographics included patient age, sex, race, insurance status, and median household income. The categorical age variable was defined using age groups $0-10,11-14$, and 15-17. Race was defined dichotomously as white vs nonwhite due to the limited number of patients in each of the more specific race categories defined by HCUP. Primary insurance status was recoded as Medicaid or private insurance. Median household income was defined by quartiles because the specific income brackets vary by year. Hospital characteristics included hospital bed size (small, medium, and large), teaching status (nonteaching vs teaching), and location of hospital (rural vs urban). We assessed concomitant medical comorbidities using the methodology described by Elixhauser. ${ }^{10}$ Due to the low incidence of many of the comorbidities, we created an index variable by designating 1 point for each comorbidity. Parameters pertaining to operative management included fusion approach, fusion level, blood loss, use of bone morphogenetic protein (BMP), use/harvest of autograft, and use of intraoperative neurophysiological monitoring (IONM). A distinction was made between the posterior-only spinal fusion (PSF) approach (ICD-9-CM codes 81.03, 81.05, 81.07, and 81.08) and a combined anterior release/fusion (AR) and posterior fusion (AR/PSF, combination of posterior ICD-9-CM codes 81.03, 81.05, $81.07-81.08$ and 1 of the anterior fusion codes 81.02, 81.04 , or 81.06). Fusion level was defined by ICD-9-CM procedure codes 81.63 (fusion or refusion of 4-8 vertebrae) and 81.64 (fusion or refusion of 9 or more vertebrae). Blood transfusion (ICD-9-CM codes 99.00, 99.02, and 99.04), the insertion of BMP (code 84.52), the use/ harvest of autograft (codes 77.00, 77.71, and 77.79), and the utilization of IONM (code 00.94) during surgery were identified using ICD-9-CM procedure codes. ${ }^{17,44}$ In this study, the variable "blood transfusion" included pre-do- 


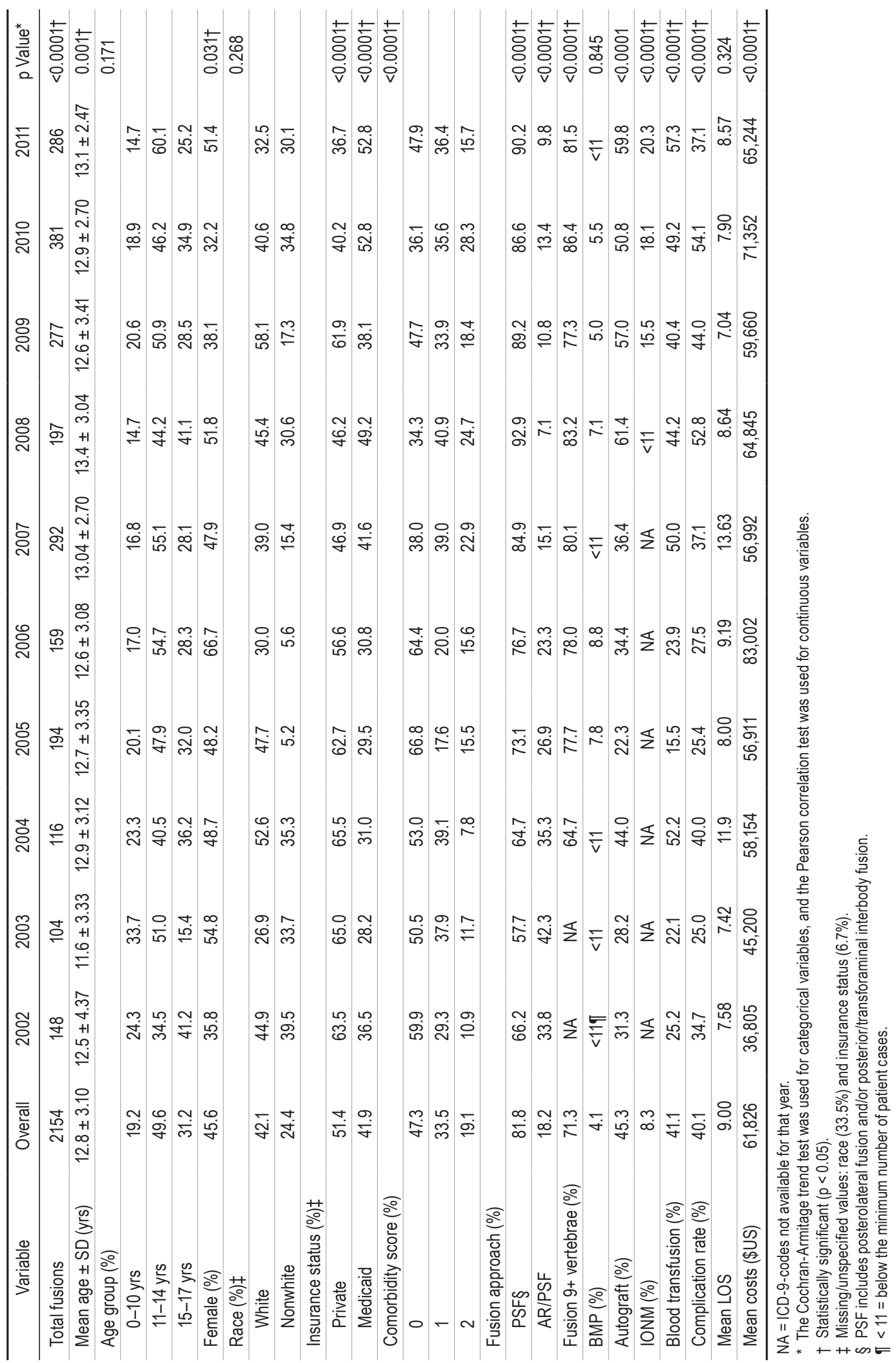


TABLE 2. In-hospital complications and outcomes in spinal fusion surgery for pediatric NMS (2002-2011)*

\begin{tabular}{|c|c|c|c|}
\hline Variable & $\begin{array}{c}\text { Complication } \\
\text { Rate (\%) }\end{array}$ & $\begin{array}{c}\text { Mean LOS in } \\
\text { Days (SD) }\end{array}$ & $\begin{array}{c}\text { Mean Total } \\
\text { Costs in \$ (SD) }\end{array}$ \\
\hline Overall & 40.1 & $9.00(14.9)$ & 61,826 \\
\hline \multicolumn{4}{|l|}{ Age group (yrs) } \\
\hline $0-10$ & 46.6 & $8.30(6.88)$ & $55,250(35,688)$ \\
\hline $11-14$ & 40.3 & $9.31(19.2)$ & $63,379(45,064)$ \\
\hline $15-17$ & 35.7 & $8.94(9.72)$ & $63,231(38,675)$ \\
\hline$p$ value & $0.002 \uparrow$ & 0.503 & $0.003 \dagger$ \\
\hline \multicolumn{4}{|l|}{ Sex } \\
\hline Male & 40.1 & $9.26(18.17)$ & $59,880(34,082)$ \\
\hline Female & 40.1 & $8.69(9.51)$ & $64,101(48,883)$ \\
\hline$p$ value & 0.973 & 0.345 & $0.026 \dagger$ \\
\hline \multicolumn{4}{|l|}{ Racef } \\
\hline White & 36.2 & 8.24 & 61,465 \\
\hline Nonwhite & 48.6 & 10.04 & 60,021 \\
\hline$p$ value & $<0.0001 \dagger$ & $0.001 \uparrow$ & 0.547 \\
\hline \multicolumn{4}{|l|}{ Insurance status $\ddagger$} \\
\hline Private & 39.5 & $9.24(18.68)$ & 61,791 (37334) \\
\hline Medicaid & 41.1 & $8.81(9.35)$ & $61,318(40,592)$ \\
\hline$p$ value & 0.697 & 0.717 & 0.595 \\
\hline \multicolumn{4}{|c|}{$\begin{array}{l}\text { Median household } \\
\text { income percentilef }\end{array}$} \\
\hline $0-25$ & 35.3 & $8.63(10.41)$ & $55,447(37,002)$ \\
\hline $26-50$ & 32.9 & $6.89(4.37)$ & $54,210(25,059)$ \\
\hline $51-75$ & 43.1 & $8.50(6.22)$ & $67,483(40,191)$ \\
\hline $76-100$ & 51.3 & $11.87(26.98)$ & $71,177(57,227)$ \\
\hline$p$ value & $<0.0001 \dagger$ & $<0.0001 \dagger$ & $<0.0001 \dagger$ \\
\hline \multicolumn{4}{|l|}{$\begin{array}{l}\text { Hospital teaching } \\
\text { status } \ddagger\end{array}$} \\
\hline Nonteaching & 27.0 & $6.33(2.63)$ & $50,903(22,666)$ \\
\hline Teaching & 41.0 & $9.16(15.47)$ & $62,280(42,484)$ \\
\hline$p$ value & $<0.0001 \dagger$ & $<0.0001 \dagger$ & $<0.0001 \dagger$ \\
\hline \multicolumn{4}{|l|}{ Comorbidity score } \\
\hline 0 & 30.5 & $6.85(4.21)$ & $54,675(33,215)$ \\
\hline 1 & 44.5 & $9.69(8.34)$ & $66,742(41,264)$ \\
\hline $2+$ & 55.8 & $13.13(31.02)$ & $70,140(54,925)$ \\
\hline$p$ value & $<0.0001 \dagger$ & $<0.0001 \dagger$ & $<0.0001 \dagger$ \\
\hline \multicolumn{4}{|l|}{ Fusion approach } \\
\hline PSF & 37.9 & $8.48(15.36)$ & $59,083(40,763)$ \\
\hline AR/PSF & 40.5 & $11.36(12.09)$ & $74,049(42,994)$ \\
\hline$p$ value & 0.330 & $<0.0001 \dagger$ & $<0.0001 \dagger$ \\
\hline \multicolumn{4}{|c|}{ Fusion 9+ vertebrae } \\
\hline No & 34.2 & $8.19(7.15)$ & $51,243(29,936)$ \\
\hline Yes & 42.4 & $9.33(17.00)$ & $65,945(44,640)$ \\
\hline$p$ value & $<0.0001 \dagger$ & $0.031 \dagger$ & $<0.0001 \uparrow$ \\
\hline \multicolumn{4}{|l|}{ BMP } \\
\hline No & 40.3 & $9.07(15.13)$ & $62,050(42,164)$ \\
\hline Yes & 33.3 & $7.43(5.09)$ & $55,128(14,611)$ \\
\hline$p$ value & 0.191 & 0.011 & $0.001 \dagger$ \\
\hline
\end{tabular}

CONTINUED IN NEXT COLUMN
» CONTINUED FROM PREVIOUS COLUMN

TABLE 2. In-hospital complications and outcomes in spinal fusion surgery for pediatric NMS (2002-2011)*

\begin{tabular}{|c|c|c|c|}
\hline Variable & $\begin{array}{l}\text { Complication } \\
\text { Rate (\%) }\end{array}$ & $\begin{array}{c}\text { Mean LOS in } \\
\text { Days (SD) }\end{array}$ & $\begin{array}{c}\text { Mean Total } \\
\text { Costs in \$ (SD) }\end{array}$ \\
\hline \multicolumn{4}{|l|}{$\begin{array}{l}\text { Use/harvest of } \\
\text { autograft }\end{array}$} \\
\hline No & 42.6 & $9.40(19.4)$ & $61,977(46,652)$ \\
\hline Yes & 37.0 & $8.52(5.6)$ & $61,646(34,513)$ \\
\hline$p$ value & $0.008 \dagger$ & 0.141 & 0.854 \\
\hline \multicolumn{4}{|c|}{ IONM (2007-2011) } \\
\hline No & 40.7 & $9.21(15.48)$ & 61,462 \\
\hline Yes & 33.1 & $6.70(2.56)$ & 65,649 \\
\hline$p$ value & $0.049 \dagger$ & $<0.0001 \dagger$ & 0.157 \\
\hline \multicolumn{4}{|c|}{ Blood transfusion } \\
\hline No & 36.6 & 8.04 & $8.04(8.19)$ \\
\hline Yes & 45.0 & 10.37 & $10.37(20.94)$ \\
\hline$p$ value & $<0.0001 \dagger$ & $0.002 \dagger$ & 0.992 \\
\hline \multicolumn{4}{|c|}{$\begin{array}{l}\text { * } p \text { values determined by the Pearson chi-square test (categorical by categori- } \\
\text { cal), independent samples t-test (dichotomous by continuous), and 1-way } \\
\text { ANOVA (categorical by continuous). } \\
\dagger \text { Statistically significant }(p<0.05) \text {. } \\
\text { † Missing/unspecified values: race }(33.5 \%) \text {, insurance status }(6.7 \%) \text {, median } \\
\text { household income }(2.18 \%) \text {, and teaching status }(1.02 \%) \text {. }\end{array}$} \\
\hline
\end{tabular}

nated autologous blood transfusion (99.02), perioperative autologous blood transfusion (99.00), and allogenic blood transfusion (99.04). The ICD-9-CM procedure code for IONM was introduced in 2007 , so trends in its utilization were unavailable for prior years.

\section{Outcome Variables}

The primary in-hospital outcomes included complications, hospital length of stay (LOS), and total hospital costs. Complications were defined using ICD-9-CM codes: respiratory complications $(518.4,518.5,518.81-$ 518.84 , and 518.89), postoperative hematoma or hemorrhage (998.1-998.13), renal and urinary complications (584 and 997.5), cardiac complications (997.1 and 410), neurological complications (997.00-997.09), thromboembolic complications $(415,415.11-415.19,451.0-451.9$, and 453.0-453.9), surgical site infection (998.5, 998.51, and 998.59), wound dehiscence (998.3, 998.31, and 998.32), implant-related complications (996.40 and 996.49), incidental durotomy $(349.3,349.31$, and 349.39), and paralytic ileus (560.1). ${ }^{28,30,32,34,44}$ The NIS database contains a variable that represents total hospital "charges," which must be differentiated from total hospital "costs." The total hospital charges variable only reflects the amount that hospitals billed for services but not the actual amount the hospitals received in payment. However, HCUP provides cost-tocharge ratio files that allow us to convert total hospital charges to total hospital costs (http://www.hcup-us.ahrq. gov/db/state/costtocharge.jsp). An Internet-based inflation calculator from the Bureau of Labor Statistics was used to adjust the costs to the current 2011 US dollar. 


\section{Statistical Analysis}

Descriptive statistics were collected for all patients undergoing spinal fusion surgery for NMS. We analyzed trends over time by utilizing the Cochran-Armitage trend test for categorical variables and 1-way ANOVA for continuous variables. The number of spinal fusion procedures performed over time was analyzed using univariate ANOVA. The impact of demographics, hospital characteristics, comorbidities, and operative characteristics on complication rate, LOS, and total hospital costs were investigated in both univariate and multivariable analyses. The Pearson chi-square test (categorical by categorical), independent samples t-test (dichotomous by continuous), and 1-way ANOVA test (categorical by continuous) were used for univariate analysis. Multivariable binary logistic regression was used to identify independent predictors of complication occurrence ( 1 or more complications), prolonged LOS (at or above the 90th percentile for LOS, 14 days), and increased total costs (at or above the 90th percentile for costs, \$103,193). All factors with p values $<0.2$ in the univariate analysis were entered into models, and factors with $\mathrm{p}$ values $<0.1$ were retained. All statistical analyses used SPSS (version 23, IBM Inc.). A p value $<0.05$ was considered statistically significant.

\section{Results \\ Patient Characteristics}

In total, we identified 2154 patients age 17 years and under who underwent spinal fusion surgery for NMS. The overall mean age of the patients was $12.8 \pm 3.10$ years (Table 1). No patients were reported in the NIS younger than 2 years of age. The study population was $45.6 \%$ female and $63.3 \%$ were white. The percentage of patients with Medicaid undergoing surgery was $41.9 \%$ overall, increasing from $36.5 \%$ in 2002 to $52.8 \%$ in 2011 ( $p<0.0001)$. Of all surgical procedures, $58 \%$ were performed at large bed size hospitals, $92.4 \%$ were performed at teaching hospitals, and $98.3 \%$ were performed at hospitals in an urban location. The proportion of patients with 1 or more Elixhauser comorbidities increased significantly from $40.2 \%$ to $52.1 \%$ over the study period ( $\mathrm{p}<0.0001$ ). Table 1 provides detailed information regarding trends in preoperative characteristics, operative management, complications, and in-hospital outcomes in pediatric patients who underwent spinal fusion for correction of NMS.

\section{Changes Over Time}

Over the 2002-2011 study period, the volume of spinal fusion procedures increased 93\% from 148 in 2002 to 286 in 2011 ( $\mathrm{p}<0.0001$; Table 1). During this same time frame we observed a significant increase in the relative number of PSF cases and a decline in the AR/PSF cases (Fig. 1). The number of PSF procedures performed increased significantly from $66.2 \%$ in 2002 to $90.2 \%$ in 2011 ( $\mathrm{p}<0.0001$ ). In contrast, the number of AR/PSF procedures decreased commensurately from $33.8 \%$ in 2002 to $9.8 \%$ in 2011 ( $\mathrm{p}<0.0001)$. Meanwhile, the number of spinal segments fused also increased with time: $64.7 \%$ underwent a $>8$-level fusion in 2004, while $81.5 \%$ underwent these longer fusions in $2011(\mathrm{p}<0.0001)$. The overall

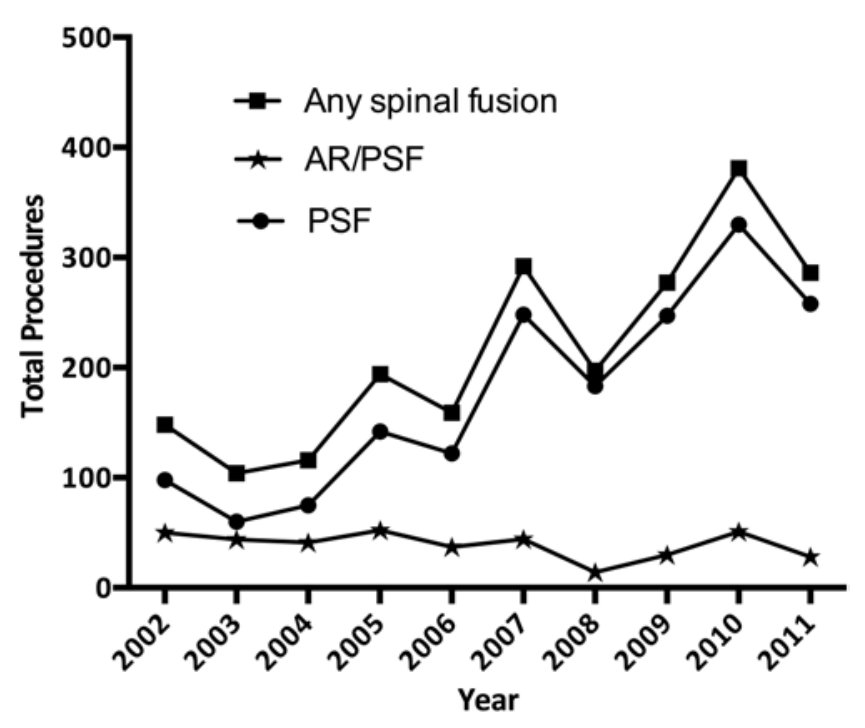

FIG. 1. Graph of the current trends in total surgical volume and surgical approach for spinal fusion surgery in pediatric NMS. Data obtained from the NIS (2002-2011).

utilization of BMP was $4.1 \%$ and fluctuated over time, but a significant trend was not demonstrated $(p=0.845)$. Since the inception of an ICD-9-CM code for IONM in 2007, its utilization increased from fewer than 11 cases in 2008 to $20.3 \%$ of cases in 2011 ( $p<0.0001$ ). The proportion of patients receiving a blood transfusion during surgery increased from $25.2 \%$ in 2002 to $57.3 \%$ in 2011 ( $p<0.0001$ ).

\section{Complications}

The overall complication rate associated with the surgery was $40.1 \%$. The top categories included pulmonary complications (28.2\%), paralytic ileus (5.6\%), postoperative hematoma or hemorrhage (4.5\%), surgical site infections $(4.0 \%)$, cardiac complications $(1.8 \%)$, neurological complications $(1.8 \%)$, wound dehiscence $(1.6 \%)$, incidental durotomy (1.1\%), renal complications $(0.93 \%)$, and implant-related complications $(0.70 \%)$. The significant trend in complication rate over time was due to fluctuations over the decade rather than a change in complication rate from $2002(34.7 \%)$ to $2011(37.1 \%)$. The overall mean LOS in this study was 9.00 days without a significant change over time $(\mathrm{p}=0.324)$. The mean total hospital costs per hospital visit was $\$ 61,826$ and demonstrated a significant increase over time from $\$ 36,805$ in 2002 to $\$ 65,244$ in 2011 (p < $0.0001)$.

\section{Univariate and Multivariable Analysis}

The univariate analysis was performed primarily to select variables for inclusion in the multivariable analysis. The results of the univariate analysis are presented in Table 2. The multivariable analysis was performed using 3 separate models with different dichotomous outcome variables (complication occurrence, prolonged LOS, and increased hospital costs).

In the first multivariable model, independent predictors of complication occurrence included non-white race 
(odds ratio [OR] 1.988, 95\% confidence interval [CI] $1.541-2.565, \mathrm{p}<0.001$, highest median household income quartile (OR 3.462, 95\% CI 2.441-4.908, p < 0.0001), 2 or more preexisting comorbidities (OR 3.226, 95\% CI 2.348$4.433, \mathrm{p}<0.001$ ), long-segment fusions (OR 1.323, 95\% CI $1.005-1.741, \mathrm{p}=0.046$ ), and use of blood transfusion (OR $1.762,95 \%$ CI $1.378-2.253, \mathrm{p}<0.0001)$.

In the second multivariable model, independent predictors of prolonged LOS included highest median household income quartile (OR 2.901, 95\% CI 1.808-4.653, p $<0.0001$ ), 2 or more preexisting comorbidities (OR 3.785, $95 \%$ CI 2.255-6.355, $\mathrm{p}<0.0001$ ), AR/PSF procedure (OR $5.082,95 \%$ CI 3.304-7.815, $\mathrm{p}<0.0001)$, and long-segment fusions (OR 2.162, 95\% CI 1.372-3.406, $\mathrm{p}=0.001$ ).

In the third multivariable model, independent predictors of increased hospital costs included age group 15-17 (OR 6.528, 95\% CI 3.246-13.126, p < 0.0001), female sex (OR 4.171, 95\% CI 2.641-6.588, $\mathrm{p}<0.0001$ ), Medicaid insurance status (OR 1.769, 95\% CI 1.097-2.853, $\mathrm{p}=0.019)$, highest median household income quartile (OR 2.787, 95\% CI 1.579-4.919, $\mathrm{p}<0.0001$ ), AR/PSF procedure (OR $11.329,95 \%$ CI $6.452-19.892, \mathrm{p}<0.0001)$, long-segment fusion (OR 3.967, 95\% CI 2.217-7.098, $\mathrm{p}<0.0001$ ), and blood transfusion (OR 2.095, 95\% CI 1.352-3.245, $\mathrm{p}=$ 0.001 ).

The use/harvest of autograft was associated with a lower likelihood of complication occurrence and a lower likelihood of increased costs (OR $0.473,95 \%$ CI $0.307-$ $0.727, \mathrm{p}=0.001)$. The use of IONM was associated with a lower likelihood of prolonged LOS (OR 0.331, 95\% CI $0.123-0.895, \mathrm{p}=0.029$ ).

\section{Discussion}

This is the largest and most recent study to date analyzing spinal fusion surgery in pediatric patients with NMS. In this study, we used data collected from the NIS (2002-2011) to examine current trends in the surgical management of NMS and to determine the incidence and identify the risk factors of postoperative complications and in-hospital outcomes. According to our findings, the volume of spinal fusion surgery for NMS increased nearly 2 -fold within the past decade, with a trend toward more all-posterior surgery. In multivariable analysis, postoperative complication rates were impacted by race, preexisting comorbidity, and blood loss. A number of factors impacted hospital LOS and total hospital costs as well. Several of these findings merit further discussion.

\section{Trend Analysis}

Consistent with previous literature and modern trends in spinal surgery, the overall number of NMS spinal fusions performed annually in children increased significantly $(193 \%)$ in the past decade. ${ }^{6,35}$

Furthermore, our results suggest that the traditional combined AR/PSF approach has become increasingly replaced by the more cost-effective all-PSF approach. Several advances in instrumentation and surgical technology are presumed to be responsible for these trends. Elsewhere in the literature, similar trends have been observed in which increased surgeon comfort with posterior-only approaches to the spine combined with the introduction of more advanced instrumentation have resulted in decreased complication rates and blood loss and increases in patient satisfaction associated with the PSF-only approach., 7,23,25,26,41

Patients undergoing NMS surgery typically have more preexisting comorbidities than any other type of spinal deformity surgery. ${ }^{4,13,22,36,38}$ Nonetheless, the overall comorbidity burden increased in our surgically treated patient population over time, possibly indicating increasing comfort with deformity correction in more seriously ill patients with NMS, or perhaps reflecting improved coding accuracy of comorbidities.

In the past decade, questions have surrounded the use of BMP, the utilization of IONM, and the use of blood transfusions in pediatric patients undergoing spinal fusion surgeries. ${ }^{4,13,14,37}$ While the off-label utilization of BMP in pediatric spinal fusion surgeries has been reported, the literature on this topic remains controversial..$^{19,20}$ The overall utilization of BMP in our study remained relatively low and did not change significantly over time.

The utilization of IONM to monitor neural structures during spine surgeries has been reported in the literature, but its cost effectiveness continues to be a topic of debate

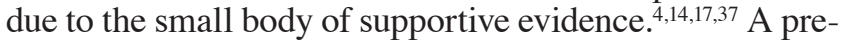
vious study by James et al. reported that IONM utilization in spine surgery increased from $1 \%$ in 2007 to $12 \%$ in 2011 , but was not associated with decreased postoperative complications. ${ }^{17}$ In our population of patients with NMS undergoing spinal fusion surgery, we report a substantial increase in the utilization of IONM between 2007 (< $1 \%)$ and $2011(20.3 \%)$. However, this observed increase in IONM use could be the result of an initial lag in ICD-9CM coding for IONM when the code was first introduced in 2007. Introduction of the code for IONM coincided with a decrease in complications and LOS, although no causal link can be made between the two. Previous literature has suggested that pediatric NMS surgeries are associated with much greater blood loss than other pediatric spinal deformity surgeries. ${ }^{15,16}$

Over the present study period, we observed a 2-fold increase in the proportion of patients receiving at least 1 blood transfusion. Furthermore, patients requiring transfusion experienced an increased complication rate and LOS. Although recent pharmacological advances such as the use of antifibrinolytics may help to decrease blood loss during surgery without necessarily increasing complications, the literature has yet to document the efficacy of these interventions in the NMS population. ${ }^{45}$

\section{Analysis of Complications}

Patient selection and surgical decision making in NMS requires careful consideration of the risks and benefits of surgery. In our study, the overall in-hospital complication rate in children undergoing NMS correction was very high $(40 \%)$ and fluctuated over the past decade. A recent meta-analysis of the literature compiled the reported rates of complications in pediatric patients surgically treated for NMS, showing pooled rates of pulmonary complications (22.71\%), implant complications (12.71\%), infections (10.91\%), neurological complications (3.01\%), and pseudarthrosis $(1.88 \%) .{ }^{38}$ Postoperative respiratory complications 
were the most commonly reported in-hospital complication (28.2\%) in this population. Respiratory assessment and management in NMS is very important because the deformity commonly impairs respiratory mechanics. ${ }^{42} \mathrm{~A}$ recent study suggested that the use of noninvasive positivepressure ventilation in NMS could reduce postoperative respiratory complications. ${ }^{18}$

Growing rods have also been shown to decrease postoperative pulmonary complications. $., 23,24,43$ The reported incidence of implant-related complications in our study was very low, as the NIS only captures complications that occur prior to the patient's discharge and does not track longitudinal outcomes. Likewise, the incidence of surgical site infection or wound dehiscence diagnosed during the primary hospitalization was $5.6 \%$ due to the lack of longitudinal follow-up data available in the NIS. A multicenter analysis of surgical site infections after instrumented spinal fusion revealed that surgery for NMS had the highest complication rate at $9.2 \% .{ }^{21}$ Our rate of reported neurological complications and incidental durotomy $(2.9 \%)$ was very similar to that in the literature (3.0\%). ${ }^{38}$

Previous literature has suggested that NMS surgery in children is associated with complicated and costly hospitalization. ${ }^{4,13,22,36,38}$ We reported average hospital LOS and total hospital costs of an average of 9 days and $\$ 61,826$ per hospitalization, respectively. According to our analysis, the occurrence of in-hospital complications increased the average LOS and total costs by $95 \%$ and $46 \%$, respectively. A study utilizing the Kids Inpatient Database (2000) determined that scoliosis surgery in children with NMS had increased hospital LOS (9.2 vs 6.1 days) and increased total hospital costs $(\$ 66,953$ vs $\$ 47,463)$ compared with children undergoing idiopathic scoliosis surgery. ${ }^{3}$ The mortality rate of children undergoing NMS correction in this study was $0.7 \%(n=15)$, and all childhood deaths were the result of an in-hospital postoperative complication. This rate was slightly higher than the mortality rate $(0.3 \%)$ reported in a previous study. ${ }^{36}$

For patient counseling and surgical decision-making purposes, awareness of patient-specific risk factors for complications and in-hospital outcomes is very important. In our study, increasing age in the pediatric population was associated with decreased complication rates and increased costs. Patients of nonwhite race were also predisposed to greater complication risks and poor in-hospital outcomes, as were patients in the highest quartile of median household income. We hypothesize that the increased likelihood of complications, prolonged LOS, and increased hospital costs in patients in the highest quartile for median income could possibly be due to a more aggressive approach to treating these patients. In addition, patients with higher comorbidities and those treated at teaching hospitals suffered from a significantly higher complication rate, LOS, and total cost of hospital visit. The adverse outcomes associated with teaching hospitals could be attributed to relatively greater severity of spinal deformity admissions at teaching hospitals, although other unknown mechanisms could also play a role. This teaching hospital effect has been observed in spine surgery literature. ${ }^{12,29,44}$ The use of BMP did not contribute to an increased likelihood of complications in this patient population. The use/ harvest of autograft was shown to increase over time and was also associated with a decreased likelihood of complications, prolonged LOS, and increased costs.

\section{Strengths and Limitations}

Despite its many strengths, the NIS database has several limitations inherent to large administrative databases. First, the NIS contains information in the form of discharge records, which depend on the sensitivity and specificity of ICD-9-CM coding practices to accurately identify diagnoses and procedures. To address this limitation, we first selected patients using procedural codes and then diagnostic codes to improve the specificity of the query. To monitor inaccuracies in the NIS database, the National Hospital Ambulatory Medical Care Survey and the American Hospital Association Annual Survey Databases are linked to the NIS to evaluate and benchmark the NIS database each year. Nonetheless, coding variability no doubt exists and contributes to inaccuracies in the data. Second, our data analyses were limited to in-hospital events, as the NIS does not track patients after they are discharged from the hospital. Consequently, our study provides an underestimation of true complication rates and costs, and does not provide any longitudinal outcome data. Long-term functional outcomes such as arthrodesis rates, returns to the operating room, degrees of scoliosis correction, or patient quality of life are not available to us in the NIS database. For this reason, coding for in-hospital complications is known to have a relatively high specificity but lower sensitivity. ${ }^{8}$ Third, we lack specifics regarding clinical presentation and physical examination, radiological evaluation, specific indications for surgery, surgeon experience or training, surgical instrumentation and technique, or exact number of spinal levels fused. All of these factors contribute to heterogeneity in our patient population and potentially reduce the validity of our analysis and conclusions.

Despite these unavoidable limitations, however, we believe that the results reported in the present study provide valuable insight for both patients and surgeons. Our study population included all cases of spinal fusion for pediatric NMS from 1 decade of discharge data from the largest publicly available inpatient care database in the US. The utilization of such a database enabled us to identify over 2000 cases of this relatively uncommon surgery. An analysis of trends in pediatric NMS has not been previously reported. The largest previously reported analysis of complications in NMS by Reames et al. examined a patient population between 2004 and 2007 and did not assess complication rates over time or after the introduction of newer instrumentation and surgical techniques ${ }^{36}$ Furthermore, this study did not discuss associations between patient demographics, comorbidities, and surgical characteristics and complication rates in pediatric NMS correction surgery. The trends reported in this study aim to serve as epidemiological proof of recent changes in the literature regarding surgical management of NMS. The awareness of risk factors for in-hospital complications and outcomes can guide patient counseling and surgical decision making. Future studies with the prospective case-control design are needed to address the limitations of the present study. 


\section{Conclusions}

In the present study, we analyzed the NIS (2002-2011) to report recent trends, complications, and outcomes in pediatric patients undergoing spinal fusion surgery for NMS. Our analysis revealed significant changes in surgical practice, such as the greater utilization of IONM and the increasing adoption of the all-PSF approach over the traditional AR/PSF approach, that were shown to decrease both complication rates and hospital LOS. However, trends toward increasing comorbidities, complication rates, blood transfusion rates, and total hospital costs may indicate the increasingly aggressive surgical management of this vulnerable patient population. The in-hospital postoperative complication rate was very high in pediatric patients undergoing spinal fusion for NMS and was detrimental to health care costs and outcomes. We identified potential risk factors for such complications to help guide preventative strategies. The results of this study inform patient counseling and surgical decision making in pediatric NMS and confirm the degree to which the surgical management of NMS has changed according to recent advancements in surgical care.

\section{References}

1. Allam AM, Schwabe AL: Neuromuscular scoliosis. PM R 5:957-963, 2013

2. Auerbach JD, Spiegel DA, Zgonis MH, Reddy SC, Drummond DS, Dormans JP, et al: The correction of pelvic obliquity in patients with cerebral palsy and neuromuscular scoliosis: is there a benefit of anterior release prior to posterior spinal arthrodesis? Spine (Phila Pa 1976) 34:E766-E774, 2009

3. Barsdorf AI, Sproule DM, Kaufmann P: Scoliosis surgery in children with neuromuscular disease: findings from the US National Inpatient Sample, 1997 to 2003. Arch Neurol 67:231-235, 2010

4. Brooks JT, Sponseller PD: What's new in the management of neuromuscular scoliosis. J Pediatr Orthop, [epub ahead of print], 2015

5. Chandran S, McCarthy J, Noonan K, Mann D, Nemeth B, Guiliani T: Early treatment of scoliosis with growing rods in children with severe spinal muscular atrophy: a preliminary report. J Pediatr Orthop 31:450-454, 2011

6. Cowan JA Jr, Dimick JB, Wainess R, Upchurch GR Jr, Chandler WF, La Marca F: Changes in the utilization of spinal fusion in the United States. Neurosurgery 59:15-20, 2006

7. Desai VR, Jea A, Gonda DD, Lam S, Luerssen TG: The effect of weekend and after-hours surgery on morbidity and mortality rates in pediatric neurosurgery. Neurosurgery 62 (Suppl 1):231, 2015 (Abstract)

8. Deyo RA, Mirza SK, Martin BI, Kreuter W, Goodman DC, Jarvik JG: Trends, major medical complications, and charges associated with surgery for lumbar spinal stenosis in older adults. JAMA 303:1259-1265, 2010

9. Dhawale AA, Shah SA, Sponseller PD, Bastrom T, Neiss G, Yorgova P, et al: Are antifibrinolytics helpful in decreasing blood loss and transfusions during spinal fusion surgery in children with cerebral palsy scoliosis? Spine (Phila Pa 1976) 37:E549-E555, 2012

10. Elixhauser A, Steiner C, Harris DR, Coffey RM: Comorbidity measures for use with administrative data. Med Care 36:8-27, 1998

11. Ferguson RL, Hansen MM, Nicholas DA, Allen BL Jr: Sameday versus staged anterior-posterior spinal surgery in a neu- romuscular scoliosis population: the evaluation of medical complications. J Pediatr Orthop 16:293-303, 1996

12. Fineberg SJ, Oglesby M, Patel AA, Pelton MA, Singh K: Outcomes of cervical spine surgery in teaching and nonteaching hospitals. Spine (Phila Pa 1976) 38:1089-1096, 2013

13. Halawi MJ, Lark RK, Fitch RD: Neuromuscular scoliosis: current concepts. Orthopedics 38:e452-e456, 2015

14. Hammett TC, Boreham B, Quraishi NA, Mehdian SMH: Intraoperative spinal cord monitoring during the surgical correction of scoliosis due to cerebral palsy and other neuromuscular disorders. Eur Spine J 22 (Suppl 1):S38-S41, 2013

15. Hassan N, Halanski M, Wincek J, Reischman D, Sanfilippo $\mathrm{D}$, Rajasekaran S, et al: Blood management in pediatric spinal deformity surgery: review of a 2 -year experience. Transfusion 51:2133-2141, 2011

16. Jain A, Njoku DB, Sponseller PD: Does patient diagnosis predict blood loss during posterior spinal fusion in children? Spine (Phila Pa 1976) 37:1683-1687, 2012

17. James WS, Rughani AI, Dumont TM: A socioeconomic analysis of intraoperative neurophysiological monitoring during spine surgery: national use, regional variation, and patient outcomes. Neurosurg Focus 37(5):E10, 2014

18. Khirani S, Bersanini C, Aubertin G, Bachy M, Vialle R, Fauroux B: Non-invasive positive pressure ventilation to facilitate the post-operative respiratory outcome of spine surgery in neuromuscular children. Eur Spine J 23 (Suppl 4):S406S411, 2014

19. Kiely PD, Cunningham ME: Off-label rhBMP-2 use in pediatric spine deformity surgery. J Neurosurg Pediatr 15:545546, 2015 (Letter)

20. Lam SK, Sayama C, Harris DA, Briceño V, Luerssen TG, Jea A: Nationwide practice patterns in the use of recombinant human bone morphogenetic protein- 2 in pediatric spine surgery as a function of patient-, hospital-, and procedure-related factors. J Neurosurg Pediatr 14:476-485, 2014

21. Mackenzie WGS, Matsumoto H, Williams BA, Corona J, Lee C, Cody SR, et al: Surgical site infection following spinal instrumentation for scoliosis: a multicenter analysis of rates, risk factors, and pathogens. J Bone Joint Surg Am 95:800806, S1-2, 2013

22. Master DL, Son-Hing JP, Poe-Kochert C, Armstrong DG, Thompson GH: Risk factors for major complications after surgery for neuromuscular scoliosis. Spine (Phila Pa 1976) 36:564-571, 2011

23. Mattila M, Jalanko T, Puisto V, Pajulo O, Helenius IJ: Hybrid versus total pedicle screw instrumentation in patients undergoing surgery for neuromuscular scoliosis: a comparative study with matched cohorts. J Bone Joint Surg Br 94:13931398,2012

24. McElroy MJ, Sponseller PD, Dattilo JR, Thompson GH, Akbarnia BA, Shah SA, et al: Growing rods for the treatment of scoliosis in children with cerebral palsy: a critical assessment. Spine (Phila Pa 1976) 37:E1504-E1510, 2012

25. Modi HN, Suh SW, Fernandez H, Yang JH, Song HR: Accuracy and safety of pedicle screw placement in neuromuscular scoliosis with free-hand technique. Eur Spine J 17:16861696, 2008

26. Modi HN, Suh SW, Song HR, Fernandez HM, Yang JH: Treatment of neuromuscular scoliosis with posterior-only pedicle screw fixation. J Orthop Surg 3:23, 2008

27. Moon ES, Nanda A, Park JO, Moon SH, Lee HM, Kim JY, et al: Pelvic obliquity in neuromuscular scoliosis: radiologic comparative results of single-stage posterior versus twostage anterior and posterior approach. Spine (Phila Pa 1976) 36:146-152, 2011

28. Murphy NA, Firth S, Jorgensen T, Young PC: Spinal surgery in children with idiopathic and neuromuscular scoliosis. What's the difference? J Pediatr Orthop 26:216-220, 2006 
29. Nandyala SV, Marquez-Lara A, Fineberg SJ, Hassanzadeh H, Singh K: Complications after lumbar spine surgery between teaching and nonteaching hospitals. Spine (Phila Pa 1976) 39:417-423, 2014

30. Nandyala SV, Marquez-Lara A, Fineberg SJ, Schmitt DR, Singh K: Comparison of perioperative outcomes and cost of spinal fusion for cervical trauma: weekday versus weekend admissions. Spine (Phila Pa 1976) 38:2178-2183, 2013

31. Niemeyer T, Freeman BJ, Grevitt MP, Webb JK: Anterior thoracoscopic surgery followed by posterior instrumentation and fusion in spinal deformity. Eur Spine J 9:499-504, 2000

32. Nuño M, Drazin DG, Acosta FLJ Jr: Differences in treatments and outcomes for idiopathic scoliosis patients treated in the United States from 1998 to 2007: impact of socioeconomic variables and ethnicity. Spine J 13:116-123, 2013

33. O'Brien T, Akmakjian J, Ogin G, Eilert R: Comparison of one-stage versus two-stage anterior/posterior spinal fusion for neuromuscular scoliosis. J Pediatr Orthop 12:610-615, 1992

34. Patil CG, Santarelli J, Lad SP, Ho C, Tian W, Boakye M: Inpatient complications, mortality, and discharge disposition after surgical correction of idiopathic scoliosis: a national perspective. Spine J 8:904-910, 2008

35. Rajaee SS, Bae HW, Kanim LEA, Delamarter RB: Spinal fusion in the United States: analysis of trends from 1998 to 2008. Spine (Phila Pa 1976) 37:67-76, 2012

36. Reames DL, Smith JS, Fu KMG, Polly DWJ Jr, Ames CP, Berven SH, et al: Complications in the surgical treatment of 19,360 cases of pediatric scoliosis: a review of the Scoliosis Research Society Morbidity and Mortality database. Spine (Phila Pa 1976) 36:1484-1491, 2011

37. Schwartz DM, Sestokas AK, Dormans JP, Vaccaro AR, Hilibrand AS, Flynn JM, et al: Transcranial electric motor evoked potential monitoring during spine surgery: is it safe? Spine (Phila Pa 1976) 36:1046-1049, 2011

38. Sharma S, Wu C, Andersen T, Wang Y, Hansen ES, Bünger $\mathrm{CE}$ : Prevalence of complications in neuromuscular scoliosis surgery: a literature meta-analysis from the past 15 years. Eur Spine J 22:1230-1249, 2013

39. Sussman MD, Little D, Alley RM, McCoig JA: Posterior instrumentation and fusion of the thoracolumbar spine for treatment of neuromuscular scoliosis. J Pediatr Orthop 16:304-313, 1996

40. Tsirikos AI, Chang WN, Dabney KW, Miller F: Comparison of one-stage versus two-stage anteroposterior spinal fusion in pediatric patients with cerebral palsy and neuromuscular scoliosis. Spine (Phila Pa 1976) 28:1300-1305, 2003

41. Tsirikos AI, Mains E: Surgical correction of spinal deformity in patients with cerebral palsy using pedicle screw instrumentation. J Spinal Disord Tech 25:401-408, 2012

42. Vialle R, Thévenin-Lemoine C, Mary P: Neuromuscular scoliosis. Orthop Traumatol Surg Res 99 (1 Suppl):S124S139, 2013

43. White KK, Song KM, Frost N, Daines BK: VEPTR ${ }^{\mathrm{TM}}$ growing rods for early-onset neuromuscular scoliosis: feasible and effective. Clin Orthop Relat Res 469:1335-1341, 2011

44. Yoshihara H, Yoneoka D: National trends in spinal fusion for pediatric patients with idiopathic scoliosis: demographics, blood transfusions, and in-hospital outcomes. Spine (Phila Pa 1976) 39:1144-1150, 2014

45. Yuan C, Zhang H, He S: Efficacy and safety of using antifibrinolytic agents in spine surgery: a meta-analysis. PLoS One 8:e82063, 2013

\section{Disclosures}

Dr. Dorward has served as a consultant to Stryker, DePuy-Synthes, Advance Medical, and Medtronic.

\section{Author Contributions}

Conception and design: Dorward. Acquisition of data: Dorward, Rumalla. Analysis and interpretation of data: Rumalla. Drafting the article: Rumalla. Critically revising the article: Dorward, Yarbrough, Pugely. Reviewed submitted version of manuscript: Dorward, Yarbrough, Pugely, Koester. Approved the final version of the manuscript on behalf of all authors: Dorward. Statistical analysis: Rumalla. Administrative/technical/material support: Koester. Study supervision: Dorward.

\section{Correspondence}

Ian Dorward, CB 8057, 4523 Clayton Ave., St. Louis, MO 63110. email: dorwardi@wudosis.wustl.edu. 